\title{
Design and Realization of Abnormal warning system of Measuring
}

\section{Equipment}

\author{
Li Shen ${ }^{1, a}$, Hua Tian ${ }^{2, b}$, Yiyi Gan ${ }^{3, c}$, Xiang Peng ${ }^{4, d}$ and Wei Qin ${ }^{5, e^{*}}$ \\ ${ }^{1,3}$ Electric Power Research Institute, State Grid Hubei Electric Power Company, Hubei, China \\ ${ }^{2,4}$ State Grid Hubei Electric Power Company, Hubei, China \\ ${ }^{5}$ Beijing VIS Energy Technology Co., Ltd., Wuhan, Hubei, China

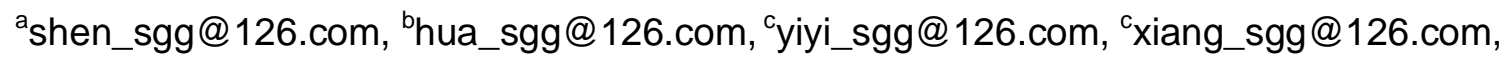 \\ eqinjordan@visenergy.cc
}

Keywords: Measuring Equipment; Dynamic Analysis of The Data; On-Line Monitoring; Early Warning Information Subscription

Abstract: Substation basic is equipped with electric energy metering system, but the electric energy metering system does not have the energy metering run abnormal alarm function. This paper introduces a measuring equipment, using the existing data of electric energy metering system by expanding calculation, realize the on-line monitoring the operation of the energy metering, analyze the acquisition anomalies and equipment failure. And according to the requirements of the users , in order to handle in time measurement circuit fault subscribe to messages in the form of alarm and alarm, mail notification and page hint.

\section{Introduction}

Measuring Equipment get data from the substation electric energy metering system, through the metering device to monitor the voltage, current loop automatically for all kinds of acquisition device, communication channel and real-time detection and abnormal alarm, application server and a number of ways to the alarm information sent to the relevant person in charge in a timely manner[1]. Provides a low cost, based on the existing threshold measuring equipment, can be in the energy metering in abnormal running timely warning technology solutions[2]. Warning system can according to the power grid structure mode, complete line loss, network loss, statistics, has the characteristics of maintainability, scalability, and realized the energy metering anomaly analysis of early warning and comprehensive information management, greatly improve the efficiency and safety of metrological work[3-4].

\section{Design Idea}

Measuring Equipment can be issued in a timely manner all kinds of measuring abnormal alarm information and events. According to the logical object classification, system alarm is divided into acquisition equipment abnormal alarm, communication faults and master station system faults three categories. Acquisition equipment mainly include watt-hour meter and terminals, which mainly includes: watt-hour meter acquisition abnormal event, phase failure, loss of flow, pressure loss not collected. Meter state abnormal event information including: fault phase sequence, battery voltage, clock error, and terminal communication interrupt; Abnormal event information terminal includes: 
restart, parameter changes, power failure, pair the main station, communication interrupt, etc. Communication including channel status anomaly, etc. Alarm host system mainly includes the current status of the main network anomalies, the running status of each server and resource usage status, as well as the main working condition of the program and so on. In addition to some abnormal collection, measurement anomaly analysis of early warning system will also be some important operation records, such as: bypass substitute, CT/FI transformation, rotation meter, battery replacement, the limit, data modification, data processing and system parameter changes.

\section{Measuring architecture of abnormal warning system}

The system logic structure. Measuring equipment is divided into the background part and the front desk business part of the two[5]. It make full use of the data provided by the mark for the electric energy metering system in substations, abnormal written warning system database, and according to the characteristics of the electric energy metering, through electric energy statistics and analysis of the various acquisition and warning events to the outside by means of fault alarm information platform, information release ways such as SMS, E-mail and Web. Measuring equipment measurement system logic structure diagram as shown in Fig.1.

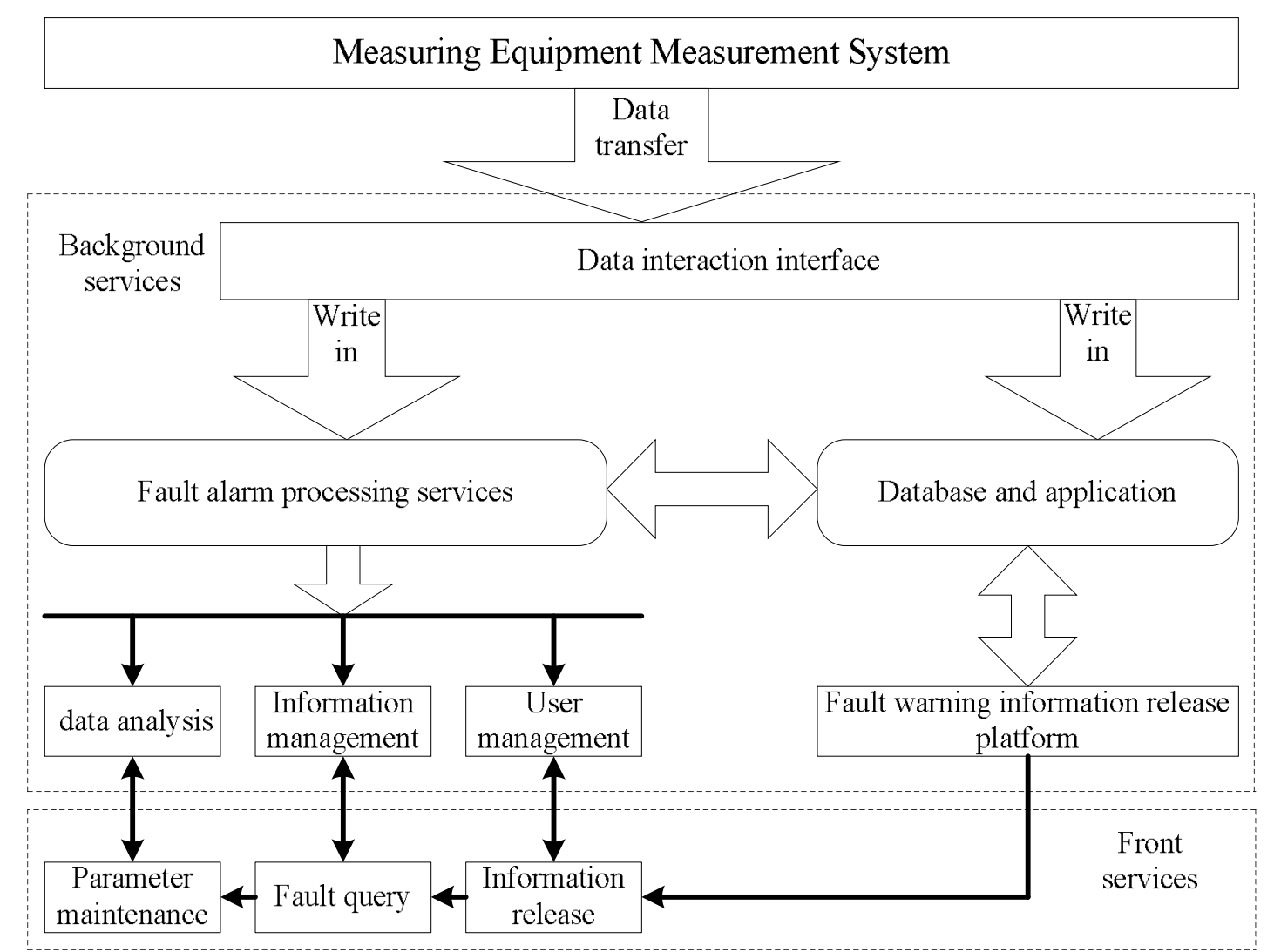

Fig.1 Measuring equipment measurement system

Background the business layer is the core of the early-warning system, it is mainly to complete the pass electric energy data synchronization, fault alarm processing services and fault alarm information release[6]. In order to guarantee the accuracy, completeness, reliability of the data, must be substation abnormal energy metering data analysis of early warning system with the energy metering system data synchronization. Fault alarm processing services mainly to complete the line loss analysis, bus balance analysis, damage analysis of the three data model analysis and statistics. When the line loss, damage and the busbar imbalance value exceeds the set threshold, the system 
will automatically generate warning events and written to the database. Fault alarm information mainly through GSM mobile SMS, E-mail or Web communication methods, such as real-time shall notify relevant alarm information management[7]. According to different managers subscribe to different event information classification strategy, triggered by the background information management module real-time related information service.

Business mainly includes the parameter maintenance, fault tracing and information release of three parts. Fault query can be classified according to time and fault query various; The alarm parameters maintenance module is mainly used to manage the alarm type, level, set the limit threshold data, event tracking time and so on. Information release business to provide users with a short message release platform of fault alarm information[8].

Early warning system and Measuring Equipment data interface implementation.Measuring equipment data is mainly composed of static data and dynamic data and static data, including network model parameters, dynamic data including the metering data, event data, abnormal alarm data, statistical analysis of data. Under normal circumstances, the static data changes little, dynamic data growth mainly depends on the dynamic data generated. Measuring equipment and mark for the power system data interface can be realized there are two kinds of technical proposal:

(1) Shared data plan

Abnormal warning system by sharing three mark for electric energy metering system database, to obtain the relevant data and information. Specific methods in order to establish the fault alarm system in the database of users, use the user in a read-only way to directly access pass electric energy data, collecting related events, abnormal alarm events, and calculation analysis data. So abnormal warning system for the measurement of the energy metering system data and event information no longer isolated storage. Abnormal warning system database stores only grid model and fault alarm information platform's own data. Abnormal warning system interactions with the data of the energy metering system interface module only complete synchronization work of power grid data model class.

(2) Data synchronization mechanism

Synchronous data is by synchronizing abnormal warning system and the measuring equipment to realize the database information way. Main synchronous information model for power grid data, the electric energy data, the collection class, abnormal alarm events, calculation and analysis data, such as synchronous data and event information need to be independent of storage.

In order to guarantee the accuracy, completeness, reliability of data, analysis and early warning must be measurement system data and error analysis of early warning system for data synchronization. Data synchronization method using data check regularly, incremental synchronous interaction patterns, namely each cycle timing check mark for the electric meter collection time scale, if changed the trigger synchronous business, and record data update at last.

(3) The data model analysis

Data analysis model including line loss analysis, bus balance analysis, damage analysis. Formula is as follows:

$$
\begin{aligned}
& L_{1}=\sum I_{a p}-\sum O_{a p} \\
& L_{1 r}=\frac{\sum I_{a p}-\sum O_{a p}}{\sum I_{a p}}
\end{aligned}
$$

In the formula, $L_{l}$ is line loss, $L a p$ is input power line, $O_{a p}$ is output power line, $L_{l r}$ is line loss rate.

The analysis of the balance of the voltage level of the substation equipment in the substation 
voltage level is calculated based on the analysis of the balance of the bus bar. Formula is as follows:

$$
B_{b r}=\frac{\sum I_{b p}-\sum O_{b p}}{\sum I_{b p}}
$$

In the formula, $B_{b r}$ is bus balance rate, $I_{b p}$ is input bus power, $O_{b p}$ is output bus power.

$$
\begin{aligned}
& V_{1}=\sum I_{p}-\sum O_{p} \\
& V_{1 r}=\frac{\sum I_{p}-\sum O_{p}}{\sum I_{p}}
\end{aligned}
$$

In the formula, $V_{l}$ is variable loss, $I_{p}$ is input power, $O_{p}$ is output power, $V_{l r}$ is variable loss rate.

\section{System function design}

System functions include data analysis, the alarm parameter maintenance, fault information query, warning information release, fault and information management, user management data interaction interface, and other functions.

Data analysis function. Measuring equipment: Data analysis function main metering data calculation and analysis, provide dynamic abnormal warning system analysis ability and the support, the main calculation analysis include:

(1) Calculate line loss, variable loss, and bus balance model according to the power grid model.

(2) Analysis measuring equipment for electric energy according to the nature of the barrier.

(3) Real time or regularly track from the calculation of measuring equipment statistics, calculation and analysis and, Vietnam limit data output state identity.

The alarm parameters maintenance functions. Alarm parameter maintenance function mainly used to manage the alarm parameters of type, level, set the limit threshold data, event tracking time and so on, the alarm types include: electric energy acquisition abnormal state information, meter event information, terminal event information, operation records, current channel status, current status, the main current program status. Alarms are divided into: error, warning, generally three categories. Data the limit threshold mainly aimed at the network loss, line loss and variable. When the corresponding data value exceeds the threshold automatically generate the alarm events.

Abnormal information query function. Fault information query function adopts $\mathrm{B} / \mathrm{S}$ mode query data, can according to the information release strategy to provide the corresponding methods of query.

The alarm information release function. The alarm information release function mainly manage the fault alarm information release. Alarm information can be classified according to the type and level of management, release, can according to need to set up SMS subscriptions, page hint, email notification, and other functions. According to the type of alarm information, level and other attribute information classification, release to related management personnel. The alarm information can be used in accordance with the authority to ensure information security.

Fault/alarm information management function.Fault alarm information management function mainly on fault alarm event information life cycle management, the system can be set up by the alarm event type, level alarm event information lifecycle, provide clean up overdue data function. System as well as artificial confirm clear the alarm information, but also the background automatically clear the alarm information. With a custom interface friendly information management strategy. 


\section{Conclusions}

This paper provides a kind of low cost, based on existing threshold billing system measuring abnormal warning system solutions, having high efficiency and practicality. Actual use of the scheme effectively solves the mark for the billing system alarm requirements, and the maintainability, reusability and extensibility than traditional threshold billing the alarm module had the very big enhancement. Measuring abnormal warning system scheme proposed in this paper, the same applies to other metering billing system and electricity information collection system, has a broad application prospect.

\section{Acknowledgements}

This work is supported by Science and Technology Projects of State Grid Hubei Electric Power Company ( Study of Intelligent online monitoring and Condition Management System of Gateway Electrical Energy Metering Device ).

\section{Reference}

[1] Yang Bai, Jian Zhang, Zhiyu Yu, CT Admittance online testing and its application [J]. Electrical Measurement and Instrumentation, 2004, 41(9): 52-55 In Chinese

[2] Shufeng Lu, The analysis and thoughts of present situation for measurement devices in gateway [J]. Electrical Measurement and Instrumentation, 2005, 42(8): 21-23 In Chinese

[3] Xiuming Zhao, Yitao Zhao, High-voltage deduced method to test errors of voltage transformers and argumentation of deduced points [J]. Electrical Measurement and Instrumentation, 2005, 42(1): 29-31 In Chinese

[4] Lei Liu, Analysis of state-monitoring for current transformer [J]. Electrical Measurement and Instrumentation, 2006, 43(9): 29-32 In Chinese

[5] Min Cao, Shibin Liang, Yi Li, Research on online monitoring technology for Yunnan power grid's electric measurement device [J]. Measurement and Instrumentation, 2007, 44(3): 41-42 In Chinese

[6] Zhikun Luo, Shengzhao Teng, Quan Wan, Remote Calibrating and monitoring system of high voltage energy measuring devices and its application [J]. Electronic Measurement Technology, 2007, 30 In Chinese

[7] Weiqin Sun, Hongcheng Li, Explain the development of Technology, 2004. Reference to a chapter in an edited book:

[8] Information on http://www.ibm.com 